\title{
Enhancement of fracture healing in the rat, modulated by compounds that stimulate inducible nitric oxide synthase
}

ACCELERATION OF FRACTURE HEALING VIA INDUCIBLE NITRIC OXIDE SYNTHASE

R. A. Rajfer,

A. Kilic,

A. S. Neviaser,

L. M. Schulte,

S. M. Hlaing,

J. Landeros,

M. G. Ferrini,

E. Ebramzadeh,

S-H. Park

Department of

Orthopaedic Surgery,

George Washington

University, Washington

DC, United States

n. A. Rajfer, MD, Resident

Physician,

A. S. Neviaser, MD, Assistant

Professor,

- L. M. Schulte, MD,

Assistant Professor,

Department of Orthopaedic

Surgery, George Washington

University, Washington DC, USA.

- A. Kilic, MD, Associate Professor,

Department of Orthopaedics,

School of Medicine, Bahcesehir

University, Istanbul, Turkey.

S. M. Hlaing, BS, Research

Associate,

- J. Landeros, BS, Research Assistant,

M. G. Ferrini, PhD, Professor

Chair, Department of Health

and Life Sciences, College of

Science and Health, Charles Drew

University of Medicine and Science,

Los Angeles, California, USA.

E. Ebramzadeh, PhD,

- S-H. Park, PhD, Professor,

Department of Orthopaedic

Surgery, The J. Vernon Luck,

Sr., M.D. Orthopaedic Research

Center, Orthopaedic Institute for

Children, University of California,

Los Angeles, California, USA.

Correspondence should be sent to S-H. Park; email: SangHyunPark@ mednet.ucla.edu

doi: $10.1302 / 2046-3758.62 . B J R-$ 2016-0164.R2

Received: 18 July 2016;

Accepted: 28 November 2016

Bone Joint Res 2017;6:90-97.

\section{Objectives}

We investigated the effects on fracture healing of two up-regulators of inducible nitric oxide synthase (iNOS) in a rat model of an open femoral osteotomy: tadalafil, a phosphodiesterase inhibitor, and the recently reported nutraceutical, COMB-4 (consisting of L-citrulline, Paullinia cupana, ginger and muira puama), given orally for either 14 or 42 days.

\section{Materials and Methods}

Unilateral femoral osteotomies were created in 58 male rats and fixed with an intramedullary compression nail. Rats were treated daily either with vehicle, tadalafil or COMB-4. Biomechanical testing of the healed fracture was performed on day 42. The volume, mineral content and bone density of the callus were measured by quantitative CT on days $\mathbf{1 4}$ and 42. Expression of iNOS was measured by immunohistochemistry.

\section{Results}

When compared with the control group, the COMB- 4 group exhibited $46 \%$ higher maximum strength ( $t$-test, $p=0.029$ ) and $92 \%$ higher stiffness ( $t$-test, $p=0.023$ ), but no significant changes were observed in the tadalafil group. At days 14 and 42, there was no significant difference between the three groups with respect to callus volume, mineral content and bone density. Expression of iNOS at day 14 was significantly higher in the COMB-4 group which, as expected, had returned to baseline levels at day 42 .

\section{Conclusion}

This study demonstrates an enhancement in fracture healing by an oral natural product known to augment iNOS expression.

Cite this article: Bone Joint Res 2017:6:90-97.

Keywords: Inducible nitric oxide synthase, Tadalafil, Fracture, Nutraceutical, Biomechanical

\section{Article focus}

- The stimulation of inducible nitric oxide synthase (iNOS) immediately post-osteotomy.

- The comparison of tadalafil and a nutraceutical in fracture healing.

\section{Key messages}

- The upregulation of iNOS may enhance fracture healing as evidenced by biomechanical properties.

- The use of nutraceuticals that upregulate iNOS may be considered as a supplement to early fracture healing.

\section{Strengths and limitations}

- The randomised design with comparative control group.

- The no dose response with either treatment arm.

- It was a small animal fracture model.

- The osteotomy created in metaphysealdiaphyseal junction.

\section{Introduction}

Fracture healing results from a complex and sequential cascade of cellular events that restore bone to its pre-fracture condition. The 
fracture site fills with a haematoma that is rich in mesenchymal cells and cytokines. This represents the inflammatory phase, and it is eventually followed by a reparative phase in which soft callus is laid down, followed by a remodeling phase. ${ }^{1}$ Although the exact regulatory mechanisms for these phases are yet to be fully understood, neovascularity, as well as osteoblast recruitment at the callus site, appears to play a key role for successful healing. ${ }^{2,3}$

Neovascularity and osteoblastic recruitment are known effects of nitric oxide (NO), which appears to be involved in the fracture healing process. ${ }^{4,5} \mathrm{NO}$ is produced by one of three isoforms of nitric oxide synthase (NOS) depending on tissue location and physiological function. 6,7 While all three of these NOS isoforms are involved in the fracture healing process, ${ }^{8-10}$ inducible NOS (iNOS) appears to be the primary isoform involved in the stimulation of osteoblasts by NO. ${ }^{11,12}$ Furthermore, when the iNOS gene is selectively deleted, fracture healing is impaired. This can be reversed by treatment with the iNOS gene. ${ }^{13}$

The effects of NO are normally mediated via its second messenger, cyclic guanosine monophosphate (cGMP), ${ }^{14,15}$ which is regulated by intracellular phosphodiesterase (PDE). ${ }^{16}$ Inhibitors of PDE, such as tadalafil, are known to increase intracellular CGMP and have been shown to accelerate early fracture healing in mice. ${ }^{17,18}$ Recently, Ferrini et $\mathrm{al}^{19}$ reported that a combination of the four herbal compounds, L-citrulline, ginger, Paullinia cupana, and muira puama (COMB-4), originally conceived as an anti-fibrotic compound, appeared in an in vivo study to produce a marked stimulation of the iNOS enzyme in rats.

We hypothesise that COMB-4 is capable of stimulating iNOS to produce elevated levels of NO and, therefore, will accelerate fracture healing, as has been shown with PDE5 inhibition. ${ }^{17,18}$ Rodents have been increasingly used to study fracture healing due to their ease of handling and cost. Although there is no standard model for studying fracture healing in a rat, there are various fixation techniques and fracture models described in the literature. ${ }^{20}$ By creating a reproducible osteotomy of the femur and using an intramedullary (IM) nail that controlled both axial and rotational stability, we created a unique standardised experimental fracture healing model for a rat from which to study the effects of both tadalafil and COMB-4. The primary outcomes included the biomechanical parameters of torsional strength, stiffness, and fracture healing stages. Secondary outcomes were bone mineral density, callus volume, and iNOS expression at the fracture site.

\section{Materials and Methods}

Experimental animals and surgical procedure. A total of 58 male Sprague-Dawley rats (ten weeks old, average body weight: $344 \mathrm{~g}$ ) were obtained from Harlan Labs/Envigo (Indianapolis, Indiana). The protocol \#1-1403-279 was approved by the Charles R. Drew University Institutional Animal Care and Use Committee. Male rats were used in order to avoid the effects of the oestrous cycle (oestrogen) as a variable. The rats were housed for one week prior to experimental procedures to permit acclimatisation, and then randomly assigned to three groups. The control group $(n=19)$ received $0.2 \mathrm{ml}$ of vehicle composed of $10 \%$ dimethyl sulfoxide, peanut butter and water by retrolingual administration. The tadalafil group $(n=20)$ received $2.0 \mathrm{mg} / \mathrm{kg}$ body weight (BW) of the PDE5 inhibitor, tadalafil (Eli Lilly, Indianapolis, Indiana), mixed with the vehicle. This dose has been shown to be equivalent to a $20 \mathrm{mg} /$ day daily dose in humans. ${ }^{21}$ The COMB-4 group $(n=19)$ received a combination of the following: muira puama (45 mg/kg BW), Paullinia cupana (45 mg/kg BW), and ginger (45 mg/kg BW) which were obtained from Naturex (Hackensack, New Jersey) as well as L-citrulline (133 mg/kg BW) which was obtained from Sigma Aldrich (St Louis, Missouri). All four components were dissolved in the vehicle. The appropriate dose of each of the four components of COMB-4 for a rat was calculated based on the equivalent recommended human daily dose as previously reported. ${ }^{19}$ All animals received the vehicle once a day for the three days prior to surgery to adapt to the taste, and began receiving on a daily basis either their respectively assigned control, or tadalafil or COMB-4 post osteotomy (day 0), which was continued until the rats were killed at two or four weeks. All surgeries were performed by one of the authors (AK) who was blinded to the rats' experimental assignment. Anaesthesia was induced with $3 \%$ isoflurane and maintained with $2.5 \%$ isoflurane throughout the surgery. All rats received subcutaneous injections of buprenorphine $0.05 \mathrm{mg} / \mathrm{kg} \mathrm{BW}$ for pre- and post-operative pain control.

Each rat then underwent a unilateral femoral osteotomy with internal fixation. Briefly, after shaving the skin, the rat was placed in the supine position and a longitudinal incision $1.5 \mathrm{~cm}$ long-centred over the superior patella was made just medial to the midline. The underlying fascia was incised at the medial patellar tendon border and the patella was dislocated laterally. The trochlea was opened with a $1.0 \mathrm{~mm}$ drill bit and the IM canal and greater trochanter were reamed with a 21-gauge hypodermic needle. The canal was sequentially reamed with an 18-gauge needle, and the epicondyle was further opened with a 16-gauge needle. The femoral shaft was accessed by sweeping the vastus medialis laterally. Cat claw scissors (Petco, San Diego, California) placed at the distal one-third of the femoral shaft were used to make a transverse osteotomy. A randomiser table was used to determine laterality of the osteotomy. While maintaining control of both osteotomy fragments, a titanium compression IM nail (RISystem, Davos, Switzerland) was applied in a retrograde manner, while visualising the fracture reduction (Fig. 1). The use of this type of IM device in rodents has been previously described. ${ }^{17,18}$ 


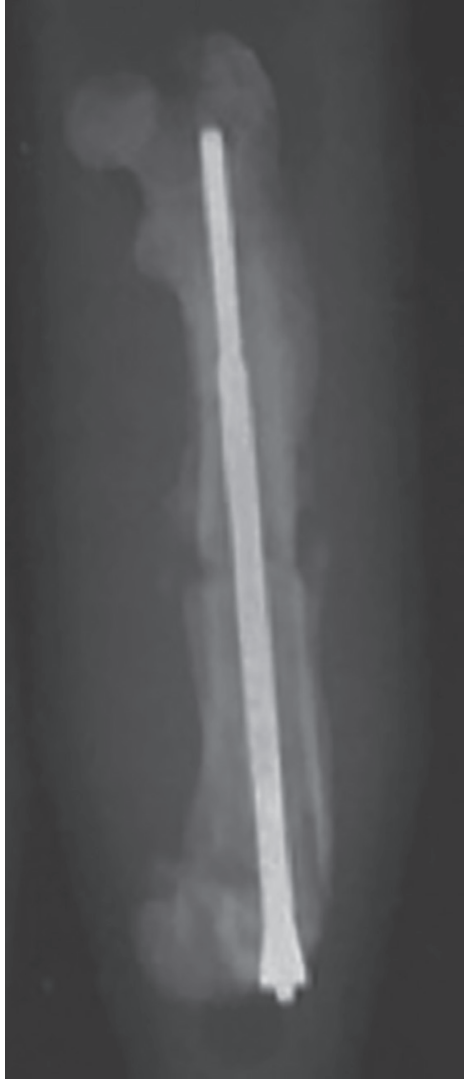

Fig. 1

Representative radiograph of a femur fixed with the intramedullary (IM) nail. The proximal end of the IM nail has a thread that permits compression of the femur fragments. This radiograph was taken after harvest at day 14

A small amount of compression was seen at the osteotomy site indicating the IM nail obtained fixation proximally at the greater trochanter and the site was tested manually for any movement.

The fascia and skin were closed in two layers using 4-0 Vicryl (Ethicon Inc., Somerville, New Jersey) and VetBond skin glue (3M, St. Paul, Minnesota). A small amount of triple antibiotic ointment (Actavis, Parsippany, New Jersey) was applied to the skin. Range of movement at the hip and knee was performed to ensure there was no penetration of the IM nail proximally and that the patella tracked appropriately. Unprotected weight-bearing was permitted immediately post-operatively, and on day 14, five rats from each of the three groups were killed using inhalational $\mathrm{CO}_{2}$ and the femurs were harvested for quantitative $\mathrm{CT}$ ( $\mathrm{qCT}$ ) and histological evaluation. On day 42 , five rats from each group were killed for the qCT and histological evaluation. The remaining 28 rats were killed for biomechanical testing.

Callus development analysis via qCT - mineral content and callus volume. A quantitative determination of callus development at days 14 and 42 was obtained with a qCT (XCT 3000, Stratec, Pforzheim, Germany). This method was selected because it allows 3D, quantitative evaluation of fracture healing, including callus development at the fracture site. Parameters were quantified as the crosssectional transverse area, bone mineral content and bone density.

After removal of the IM nail, a bony section $3 \mathrm{~mm}$ in length, that included the osteotomy site in the centre, was analysed using three consecutive transverse qCT scans of $1.1 \mathrm{~mm}$ in thickness and $0.1 \mathrm{~mm} \times 0.1 \mathrm{~mm}$ in pixel size. A total of three images were obtained; one for the bone fragment proximal to the osteotomy, one for the osteotomy site itself and one for the distal bone fragment. XCT Series software package (version 5.21, Stratec) was used to calculate the mineral content, mineralised callus area and bone mineral density of each image. Total mineral content and callus volume of the $3 \mathrm{~mm}$ segment was calculated by combining all three section values. In order to assess progression of remodeling, higher density callus ( $>500 \mathrm{mg} / \mathrm{cm}^{3}$ ) was measured and separated from total callus (> $299 \mathrm{mg} / \mathrm{cm}^{3}$ ). Mineral density greater than $850 \mathrm{mg} / \mathrm{cm}^{3}$ was considered cortical bone density and was eliminated from newly formed callus calculations. ${ }^{22}$ Biomechanical evaluation. After euthanasia at day 42 , each femur was harvested and both ends of the femur were embedded in blocks of polymethylmethacrylate after removal of the IM nail. Only a $12 \mathrm{~mm}$ long segment of the bone, which included the osteotomy in the centre, was exposed. The specimen was then secured onto a torsional testing apparatus mounted into an MTS bi-axial load frame (MTS mini-bionix 858, Minneapolis, Minnesota). Torsion to failure was applied at a rate of $12^{\circ} /$ min. $^{23}$ There were two parameters derived from the torque-displacement curve for each specimen's load to failure test: torsional stiffness and maximum torque. Additionally, using the failure pattern, each specimen was classified into one of four biomechanical stages of fracture repair as described by White et al. ${ }^{24} \mathrm{~A}$ Stage I fracture is rubbery, indicating only soft callus formation. Stages II through IV exhibit higher stiffness, indicating failure of bone with progressively higher mineralised tissue. Stage I and II fractures fail through the original osteotomy line. Stage III failure occurred partially through the original osteotomy line and partially through intact bone, while Stage IV failure occurred entirely through intact bone.

Immunohistochemical expression of iNOS. After fixation in $4 \% \mathrm{p}$-formaldehyde, femurs from both days 14 and 42 were decalcified in 10\% formic acid for five days. Each femur was rinsed in PBS, both epiphyses were removed and the shaft was cut longitudinally in two halves. Both halves were processed for paraffin embedding sections. Five-micrometer paraffin embedded sections were employed for immunostaining with polyclonal antibody (dilution 1:250) against iNOS (BD Pharmingen, San Diego, California). Next, the sections were then incubated with biotinylated anti-Rabbit IgG, respectively, followed by $A B C$ complex (Vector labs, Temecula, California) and 3,3'diaminobenzidine (Sigma Aldrich) slides were counter-stained with a 
Table I. Newly formed total and hard callus at the osteotomy site at days 14 and 42 (presented as mean and standard error of the mean)

\begin{tabular}{|c|c|c|c|c|c|c|}
\hline \multirow[b]{2}{*}{ Group } & \multicolumn{2}{|c|}{ Callus volume $\left(\mathrm{mm}^{3}\right)$} & \multicolumn{2}{|c|}{ Mineral content (mg) } & \multicolumn{2}{|c|}{ Bone density $\left(\mathrm{mg} / \mathrm{cm}^{3}\right)$} \\
\hline & Day 14 & Day 42 & Day 14 & Day 42 & Day 14 & Day 42 \\
\hline \multicolumn{7}{|c|}{ Newly formed total callus (> $299 \mathrm{mg} / \mathrm{cm}^{3}$ ) } \\
\hline Control & $44.0(4.5)$ & $64.0(9.0)$ & $19.7(2.4)$ & $33.0(5.1)$ & $445.8(11.6)$ & $512.2(8.1)$ \\
\hline Tadalafil & $44.9(5.8)$ & $66.3(10.1)$ & $20.8(3.2)$ & $34.1(5.7)$ & $458.4(14.6)$ & $510.0(8.5)$ \\
\hline COMB-4 & $37.3(3.9)$ & $63.2(4.8)$ & $16.4(1.9)$ & $31.7(2.8)$ & $438.4(4.5)$ & $500.0(8.9)$ \\
\hline \multicolumn{7}{|c|}{ Newly formed hard callus ( $>500 \mathrm{mg} / \mathrm{cm}^{3}$ ) } \\
\hline Control & $15.1(2.2)$ & $35.7(5.5)$ & $10.0(1.4)$ & $24.6(3.6)$ & $659(4.7)$ & $693.2(11.9)$ \\
\hline Tadalafil & $16.4(2.4)$ & $35.6(5.6)$ & $11.0(1.6)$ & $24.1(3.7)$ & $665(1.0)$ & $697.2(5.5)$ \\
\hline COMB-4 & $13.3(1.4)$ & $33.4(3.5)$ & $8.8(0.9)$ & $22.8(2.4)$ & $661(4.4)$ & $683.7(2.5)$ \\
\hline
\end{tabular}

$\mathrm{n}=4$ for day 14 control and tadalafil, and day 42 COMB- 4 groups; $n=5$ for day 14 COMB- 4 and day 42 control and tadalafil groups

COMB-4: L-citrulline, Paullinia cupana, ginger and muira puama
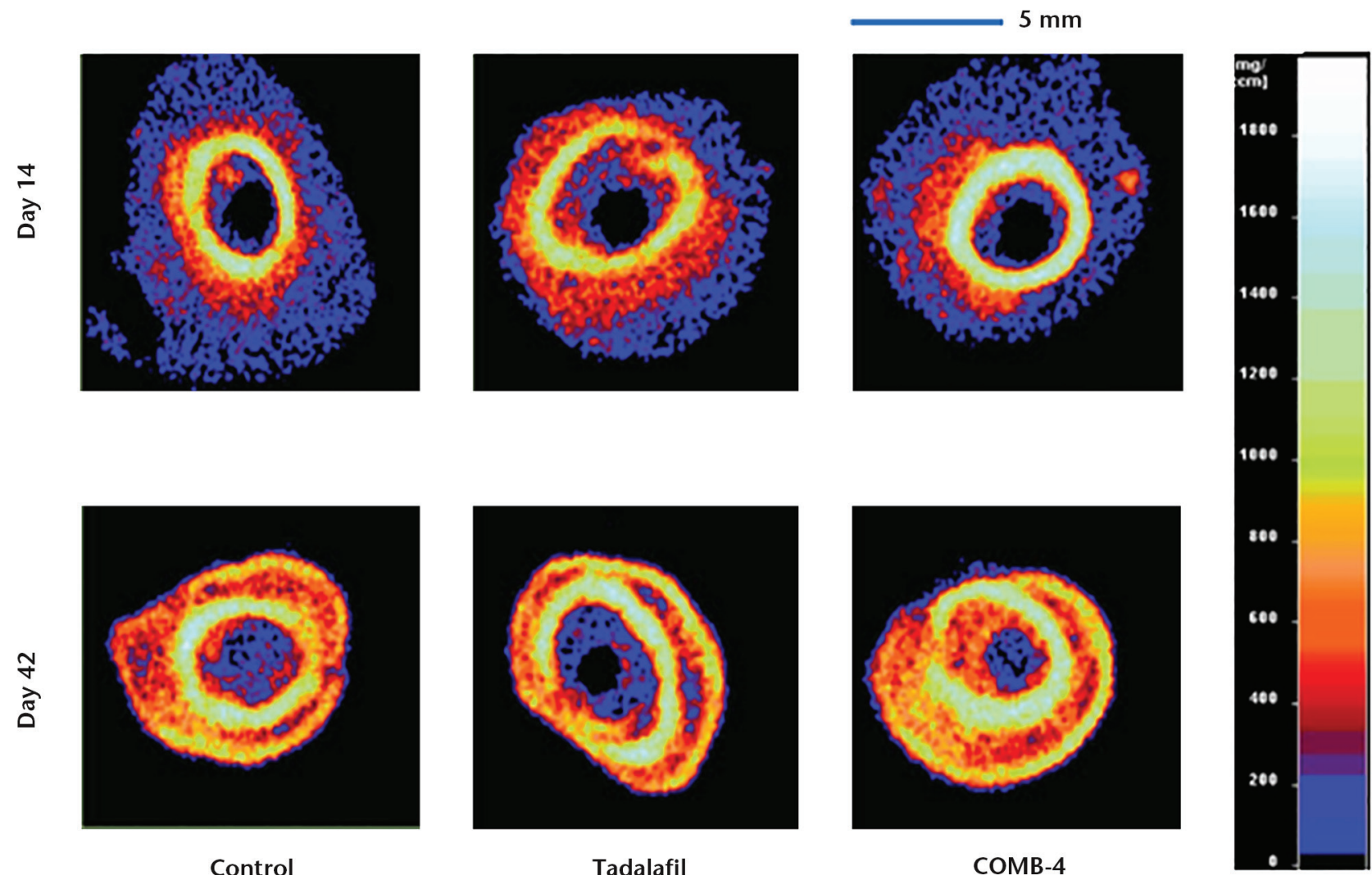

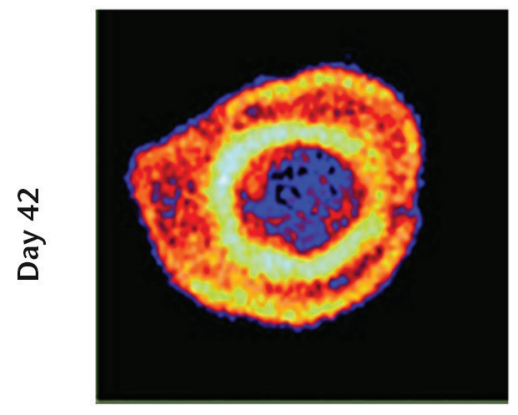

Control

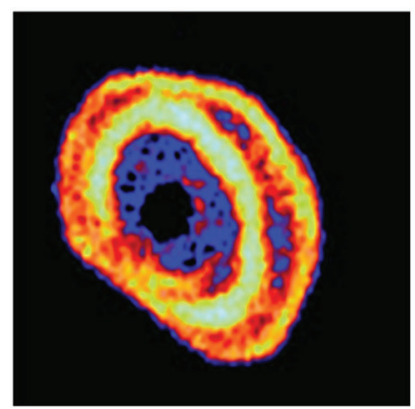

Tadalafil

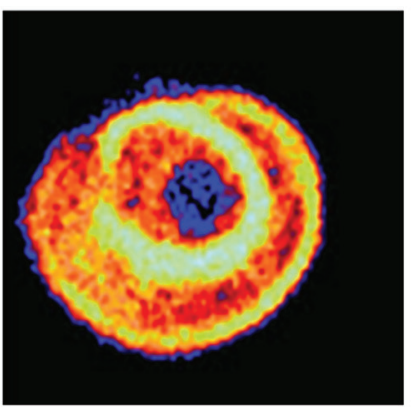

COMB-4

Fig. 2

Representative quantitative CT (qCT) images of the three groups at days 14 and 42 . These qCT images were taken $1 \mathrm{~mm}$ below the osteotomy site to eliminate bone fragment overlap at the osteotomy level. The right side bar indicates bone mineral density.

hematoxylin solution. Stained sections were dehydrated and then mounted with Permount. At least six pictures per section were taken with a Leica microscope (Leica Microsystems, Wetzlar, Germany) at $200 \times$ magnification. Integrated optical density (IOD) per cell was determined using Image Pro 7.1 software (Media Cybernetics, Silver Spring, Maryland). After images were calibrated for background lighting, IOD results are proportional to the unweighted average optical density, which is used to determine the concentration of immunoreactive antigen. Each slide assayed had its corresponding negative control. In all cases, six non-overlapping fields were screened per tissue sections and at least three sections per animal were analysed.

Statistical analysis. A one-way analysis of variance (ANOVA) was used to compare the outcome variables, including callus volume, bone mineral content, bone density, torsional stiffness and maximum torque at failure. The independent variables were days 14 and 42 and treatment type (three categories including control). Oneway ANOVA was followed by least significant difference post hoc tests to compare individual pairs of groups. Nonparametric tests were used to compare the fracture repair stages among the three groups. 
Table II. Biomechanical properties of healed fractures at day 42 (from torsion test, presented as mean and standard error of the mean)

\begin{tabular}{llll}
\hline Group & Fracture repair stage $\left(\mathbf{I}\right.$, II, III or IV) ${ }^{\dagger}$ & Maximum torque (Nmm) & Torsional stiffness (Nmm/ $\left.{ }^{\circ}\right)$ \\
\hline Control $(n=7)$ & $2.4(0.2)$ II $n=4 ;$ III $n=3 ;$ IV $n=0$ & $283.3(59.8)$ & $46.8(12.8)$ \\
Tadalafil $(n=10)$ & $2.4(0.2)$ II $n=6 ;$ III $n=4 ;$ IV $n=0$ & $326.8(50.0)$ & $59.9(10.7)$ \\
COMB-4 $(n=9)$ & $2.8(0.3)$ II $n=3 ;$ III $n=2 ;$ IV $n=4$ & $421.3(52.0)^{*} p=0.029$ \\
\hline
\end{tabular}

*compared with control using analysis of variance followed by least significant difference for multiple comparisons

tfracture repair stage numerical conversion for mean and standard error of the mean

COMB-4: L-citrulline, Paullinia cupana, ginger and muira puama

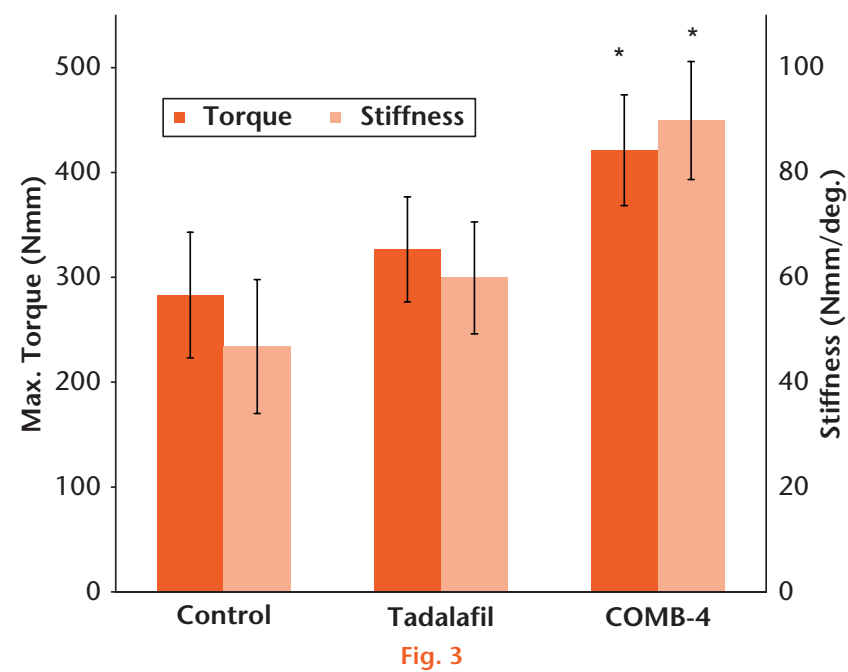

Maximum torque (strength) and stiffness of healing fracture at day 42 . Results are expressed as mean and standard error of the mean ( $p<0.05$ when compared with control group using analysis of variance followed by least significant difference for multiple comparisons).

For quantitative image analysis of the histological observations, values were expressed as the mean and standard error of the mean (SEM). The normality distribution of the data was established using the Wilk-Shapiro test. Multiple comparisons were analysed by a single factor analysis of variance, followed by post hoc comparisons with the Tukey test according to GraphPad Prism (version 5.1) for Windows (GraphPad Software, San Diego, California). A p-value of $<0.05$ was considered significant.

\section{Results}

A total of five rats (two from day 14 and three from day 42) were excluded from the study due to either an infection discovered at the osteotomy site $(n=4)$ or a comminuted fracture $(n=1)$. Therefore, only 53 animals were used for the analysis.

Callus development analysis - mineral content and callus volume. All three groups demonstrated about a $50 \%$ increase in both the mineral content and mineralised callus volume at the osteotomy site at day 42 when compared with day 14 (Table I). However, at both of these time points neither the tadalafil nor the COMB-4 group demonstrated a statistically significant difference compared with the control group. Representative

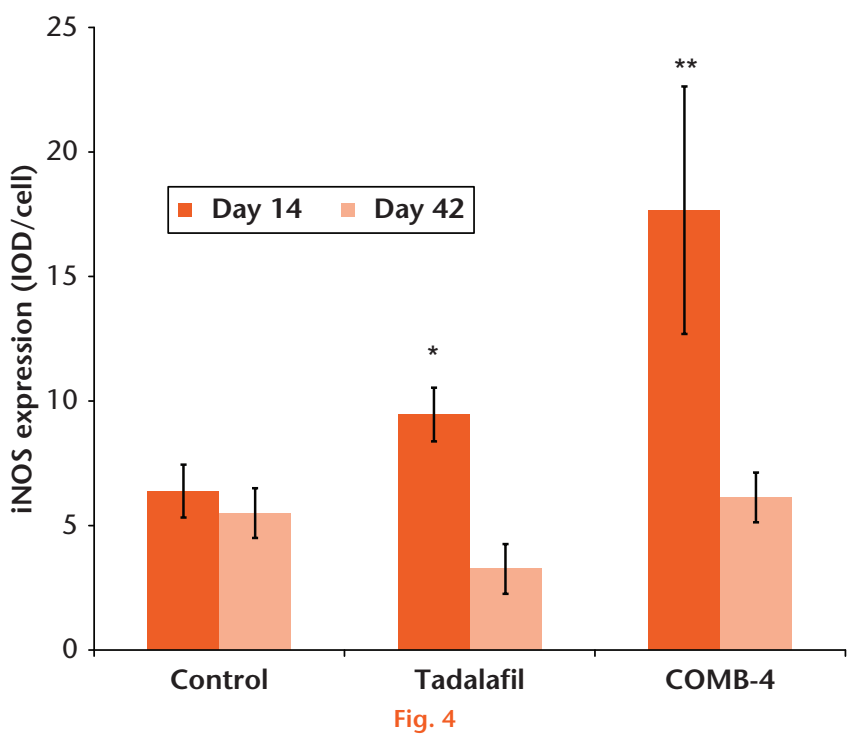

Graph showing the effect of tadalafl and COMB-4 on inducible nitric oxide synthase (iNOS) expression at day 14 and day 42 after femoral osteotomy. The graph shows quatitative image analysis results of the intensity of iNOS expression per cell. Results are expressed as mean and standard error of the mean $\left({ }^{*} \mathrm{p}=0.043 ;{ }^{* *} \mathrm{p}=0.002\right.$ when compared with control group, using analysis of variance followed by Tukey as post hoc test) (COMB-4: L-citrulline, Paullinia cupana, ginger and muira puama).

qCT images of the three experimental groups at the two different time points are shown in Figure 2.

Biomechanical properties. At day 42, the mean values for all three biomechanical parameters (maximum torque, torsional stiffness and fracture repair stage) were highest in the COMB-4 group (Table II, Fig. 3). The COMB-4 group exhibited $46 \%$ higher maximum strength $(p=0.029)$ and $92 \%$ higher stiffness $(p=0.023)$ than those of the control group. The COMB-4 group also exhibited 29\% higher maximum strength $(p=0.497)$ and $50 \%$ higher stiffness $(p=0.396)$ than that of the tadalfil group but this difference was not statistically significant. The tadalafil group exhibited $15 \%$ higher maximum strength $(p=0.125)$ and $28 \%$ higher stiffness $(p=0.076)$ with respect to the control group, but this change was not statistically significant.

All of the osteotomies were healed at day 42 and were higher than Stage II of the biomechanical classification of fracture repair. Stage IV fracture repairs were not seen in the tadalafil or in the control groups, but were seen in four rats from the COMB-4 treatment group. 


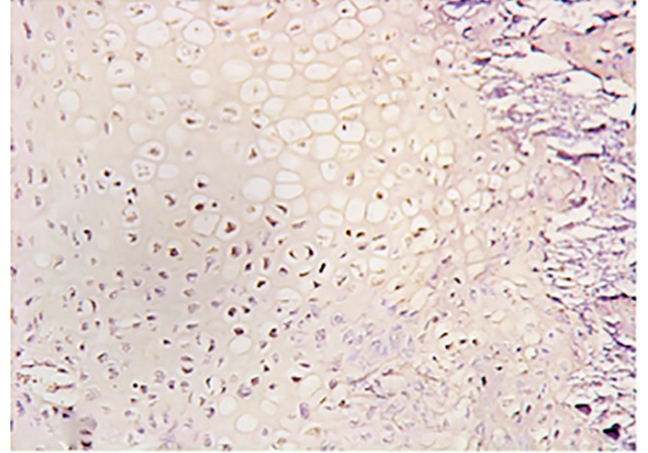

Fig. $5 \mathrm{a}$

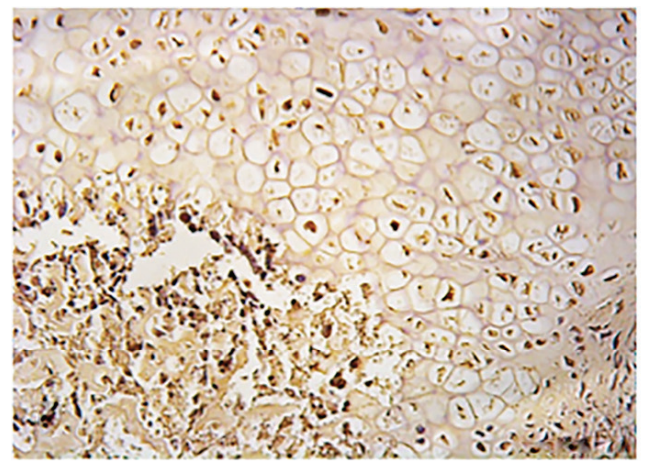

Fig. $5 \mathrm{c}$

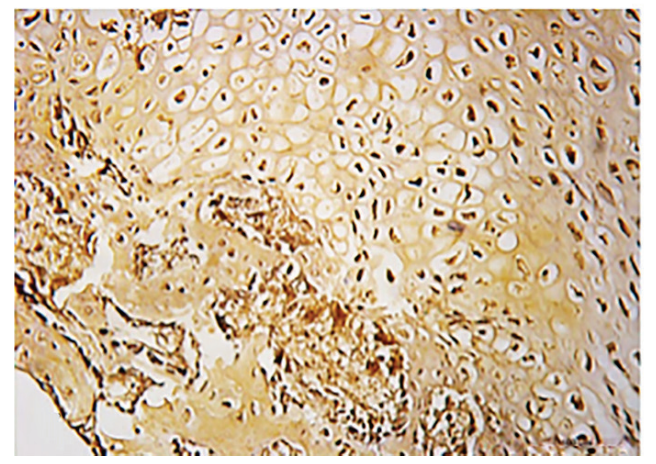

Fig. $5 \mathrm{e}$

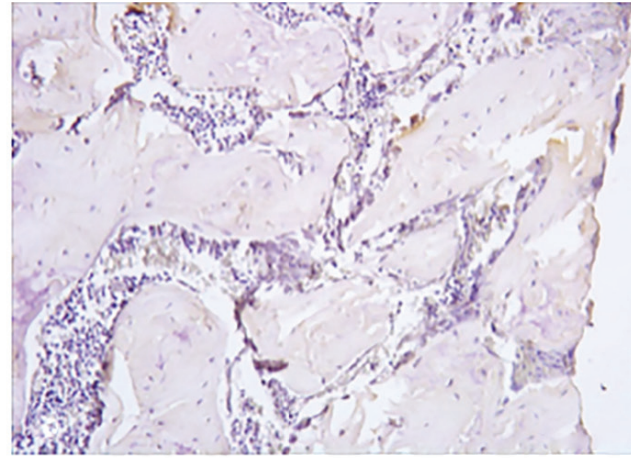

Fig. $5 b$

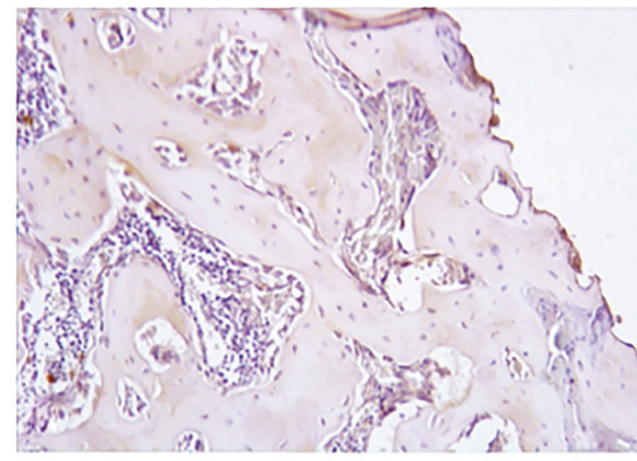

Fig. $5 d$

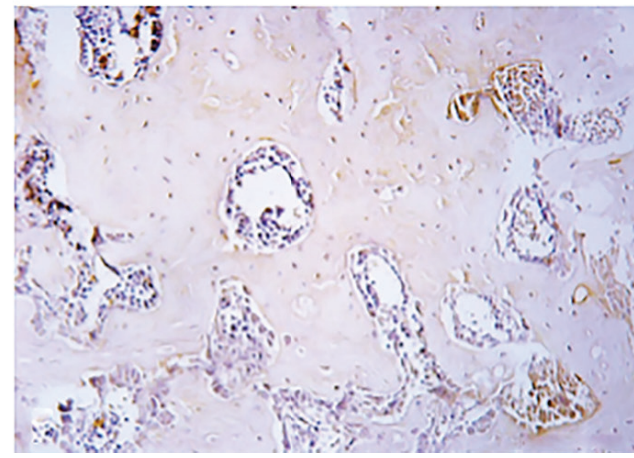

Fig. $5 f$

Effect of tadalafil and COMB-4 on inducible nitric oxide synthase (iNOS) expression at day 14 and day 42 after femoral osteotomy determined by immunohistochemistry. Microphotographs of iNOS expression taken at the osteotomy site: a) and b) are control; c) and d) are tadalafil; e) and f) are COMB-4. Panels (a), (c) and (e) depict 14-day results and panels (b), (d) and (f) depict 42-day results.

The remaining factures in the three groups were either Stage II or Stage III (Table II).

Immunohistochemical expression of iNOS. At 14 days after the osteotomy, iNOS expression measured at the callus level was significantly increased in the tadalafil group by $30 \%(9.45$ SEM 1.08, $p=0.043)$ and in the COMB-4 group by $180 \%$ (18.43 SEM 4.34, $p=0.002)$ when compared with the control group (6.38 SEM 1.06) (Fig. 4). Expression of iNOS was localised mostly in chondrocytes at the level of the cartilaginous callus and in osteoblasts at the calcification front (Figs 5a, 5c and 5f). However, as expected, iNOS expression in all three groups at day 42 had returned to baseline values (Figs $5 b, 5 d$ and $5 f$ ).

\section{Discussion}

This study was conceived to determine whether prolonged upregulation of the NO-cGMP pathway via iNOS immediately following a fracture would enhance the healing process. This hypothesis came about from the observation that $\mathrm{NO}$ is involved in fracture healing as shown by the induction of all three isoforms of NOS in a specific sequential manner during the fracture healing process; $8-10,25$ iNOS is known to be expressed in different tissues in response to an injury 26,27 and its induction in such injured tissues is considered a protective mechanism against abnormal wound healing; ${ }^{28}$ iNOS is the first NOS isoform induced immediately and rapidly following 
a fracture but then tapers off after peaking around the one- to two-week mark; ${ }^{8-10}$ and iNOS is closely involved with osteoblast function and bone formation. 11,29 Furthermore, although inhibition of all three NOS genes will result in retardation of both bone formation and normal fracture healing, $, 9,30$ it has been shown that the specific targeted inhibition or deletion of the iNOS enzyme will negatively impact the fracture healing process while replacement of the deficient iNOS gene to iNOS deficient animals will result in normal fracture healing. ${ }^{13}$

PDE inhibitors are compounds that can upregulate iNOS via the NO-cGMP pathway ${ }^{31}$ and have been reported to enhance fracture healing ${ }^{17}$ as well as promote the differentiation of osteogenic precursor cells to osteoblasts. ${ }^{32}$ However, after 42 days of daily tadalafil, fracture healing, as measured by either the biomechanical fracture repair stage, maximum torque or torsional stiffness, was not significantly enhanced when compared with the control condition.

In contrast, COMB-4, a product consisting of a predetermined mixture of the four natural constituents of L-citrulline, ginger, muira puama and Paullinia cupana, recently reported as an up-regulator of iNOS, ${ }^{19}$ significantly enhanced fracture healing as measured by biomechanical properties. In the present study, the smaller mineralised callus volume and the newly mineralised callus seen in these COMB-4 animals may be indicative of an accelerated mineralisation of bridging callus that contributes to the superior biomechanical properties of the COMB-4 group compared with both the tadalafil and control groups.

It is possible that the biomechanical findings of the tadalafil group could be attributed to the dosage of tadalafil used. The $2 \mathrm{mg} / \mathrm{kg}$ daily dosage of tadalafil used in this study was lower than the $5 \mathrm{mg} / \mathrm{kg}$ dose reported in the literature to be efficacious as an anti-fibrotic agent in the rat. ${ }^{33}$ This $5 \mathrm{mg} / \mathrm{kg}$ dose in the rat is equivalent to $40 \mathrm{mg}$ per day in a human, ${ }^{21}$ which supersedes the highest human recommended daily dose for this drug when administered for urological dysfunctions, ${ }^{34}$ but is considered to be an optimum dose for patients with pulmonary arterial hypertension. ${ }^{35}$ We speculate that the difference in effect between tadalafil and COMB-4 could be attributed to the varying dosages of tadalafil used in the abovementioned studies as well as to absorption of the tadalafil. Some of those studies used non-soluble media with ingestion of the product while others used a dissolvable solution with a differential effect of the product on stimulating iNOS.

Our study in the rat has several limitations. First, we used an unlocked IM nailing system and, as such, axial and rotational stability was not fully controlled. This may have led to some displacement of a fracture fragment due to a larger medullary canal at the distal diaphysis, where most of the osteotomies were located, such that the reduction may not have been perfect and resulted in different patterns of callus formation. ${ }^{22,36}$ It is also known that fracture callus development and size are affected by the stability of the fracture. ${ }^{37}$ Finally, as stated earlier, the dosages of tadalafil and COMB-4 were individually calculated based on previous published studies in the rat and prescribed human dosages. It is possible that these doses were not optimum for treating a fracture in this animal model because of potential pharmacokinetic differences between the human and the rat. Further investigation using different doses of both tadalafil and COMB-4, singly or in combination, may be required to elucidate the optimum dose of each product.

This study demonstrates the efficacy of a natural product in enhancing the functional strength of bone following an osteotomy in a rat. COMB-4 by itself resulted in excellent biomechanical properties associated with fracture healing when compared with that seen with either the control or tadalafil group. L-citrulline, muira puama, Paullinia cupana and ginger, the constituents of COMB4 , are known in humans to have an innocuous side effect profile. ${ }^{38,39}$ It is possible that COMB-4 may play a potential future role in enhancing the healing process associated with musculoskeletal fractures, although additional studies will certainly be required in humans to confirm its clinical efficacy.

\section{References}

1. Caplan Al. Cartilage begets bone versus endochondral myelopoiesis. Clin Orthop Relat Res 1990:257-267.

2. Hausman MR, Schaffler MB, Majeska RJ. Prevention of fracture healing in rats by an inhibitor of angiogenesis. Bone 2001;29:560-564.

3. Keramaris NC, Calori GM, Nikolaou VS, et al. Fracture vascularity and bone healing: a systematic review of the role of VEGF. Injury 2008;39:S45-S57.

4. Hikiji H, Shin WS, Oida S, et al. Direct action of nitric oxide on osteoblastic differentiation. FEBS Lett 1997;410:238-242.

5. Baldik Y, Talu U, Altinel L, et al. Bone healing regulated by nitric oxide: an experimental study in rats. Clin Orthop Relat Res 2002:343-352.

6. Bredt DS. Endogenous nitric oxide synthesis: biological functions and pathophysiology. Free Radic Res 1999;31:577-596.

7. Förstermann U, Gath I, Schwarz P, et al. Isoforms of nitric oxide synthase. Properties, cellular distribution and expressional control. Biochem Pharmacol 1995;50:1321-1332.

8. Corbett SA, Hukkanen $\mathbf{M}$, Batten $\mathbf{J}$, et al. Nitric oxide in fracture repair. Differential localisation, expression and activity of nitric oxide synthases. J Bone Joint Surg [Br] 1999;81-B:531-537.

9. Diwan AD, Wang MX, Jang $\mathbf{D}$, et al. Nitric oxide modulates fracture healing. J Bone Miner Res 2000;15:342-351.

10. Zhu W, Murrell GA, Lin J, et al. Localization of nitric oxide synthases during fracture healing. J Bone Miner Res.2002;17:1470-1477.

11. Hukkanen M, Hughes FJ, Buttery LD, et al. Cytokine-stimulated expression of inducible nitric oxide synthase by mouse, rat, and human osteoblast-like cells and its functional role in osteoblast metabolic activity. Endocrinology 1995;136:5445-5453.

12. Wimalawansa SJ. Nitric oxide and bone. Ann N Y Acad Sci 2010;1192:391-403

13. Baldik Y, Diwan AD, Appleyard RC, et al. Deletion of iNOS gene impairs mouse fracture healing. Bone 2005;37:32-36.

14. Katsuki S, Arnold W, Mittal C, Murad F. Stimulation of guanylate cyclase by sodium nitroprusside, nitroglycerin and nitric oxide in various tissue preparations and comparison to the effects of sodium azide and hydroxylamine. J Cyclic Nucleotide Res 1977;3:23-35.

15. Arnold WP, Mittal CK, Katsuki S, Murad F. Nitric oxide activates guanylate cyclase and increases guanosine 3区:5囚-cyclic monophosphate levels in various tissue preparations. Proc Natl Acad Sci U S A 1977;74:3203-3207.

16. Weiss B. Differential activation and inhibition of the multiple forms of cyclic nucleotide phosphodiesterase. Adv Cyclic Nucleotide Res 1975;5:195-211. 
17. Histing T, Marciniak K, Scheuer C, et al. Sildenafil accelerates fracture healing in mice. J Orthop Res 2011;29:867-873.

18. Toğral G, Arıkan $\mathbf{M}$, Korkusuz $\mathbf{P}$, et al. Positive effect of tadalafil, a phosphodiesterase-5 inhibitor, on fracture healing in rat femur. Eklem Hastalik Cerrahisi 2015;26:137-144.

19. Ferrini MG, Hlaing SM, Chan A, Artaza JN. Treatment with a combination of ginger, L-citrulline, muira puama and Paullinia cupana can reverse the progression of corporal smooth muscle loss, fibrosis and veno-occlusive dysfunction in the aging rat. Andrology (Los Angel) 2015;4:132.

20. Histing T, Garcia P, Holstein $\mathbf{J H}$, et al. Small animal bone healing models: standards, tips, and pitfalls results of a consensus meeting. Bone 2011;49:591-599.

21. Reagan-Shaw S, Nihal $\mathbf{M}$, Ahmad $\mathbf{N}$. Dose translation from animal to human studies revisited. FASEB J 2008;22:659-661.

22. Park SH, Silva M. Effect of intermittent pneumatic soft tissue compression of fracture healing in an animal model. J Bone Joint Surg [Am]2003;85-A:1446-1453.

23. Park SH, O'Connor K, McKellop H, Sarmiento A. The influence of active shear of compressive motion on fracture healing. J Bone and Joint Surg [Am] 1998:80-A;868-878.

24. White AA 3rd, Panjabi MM, Southwick WO. The four biomechanical stages of fracture repair. J Bone and Joint Surg [Am] 1977;59-A:188-192.

25. Klein-Nulend J, van Oers RF, Bakker AD, Bacabac RG. Nitric oxide signaling in mechanical adaptation of bone. Osteoporos Int 2014;25:1427-1437.

26. Geller DA, Billiar TR. Molecular biology of nitric oxide synthases. Cancer Metastasis Rev 1998;17:7-23.

27. Bentz BG, Simmons RL, Haines GK III, Radosevich JA. The yin and yang of nitric oxide: reflections on the physiology and pathophysiology of NO. Head Neck 2000;22:71-83.

28. Ferrini MG, Vernet D, Magee TR, et al. Antifibrotic role of inducible nitric oxide synthase. Nitric Oxide 2002;6:283-294.

29. Watanuki M, Sakai A, Sakata T, et al. Role of inducible nitric oxide synthase in skeletal adaptation to acute increases in mechanical loading. J Bone Miner Res 2002;17:1015-1025.

30. Fox SW, Chambers TJ, Chow JW. Nitric oxide is an early mediator of the increase in bone formation by mechanical stimulation. Am J Physiol.1996;270:E955-E960.

31. AhIner J, Ljusegren ME, Grundström N, Axelsson KL. Role of nitric oxide and cyclic GMP as mediators of endothelium-independent neurogenic relaxation in bovine mesenteric artery. Circ Res 1991;68:756-762.

32. Wakabayashi S, Tsutsumimoto T, Kawasaki S, et al. Involvement of phosphodiesterase isozymes in osteoblastic differentiation. J Bone Miner Res 2002;17:249-256.

33. Kovanecz I, Rambhatla A, Ferrini MG, et al. Chronic daily tadalafil prevents the corporal fibrosis and veno-occlusive dysfunction that occurs after cavernosal nerve resection. BJU Int 2008;101:203-210.
34. No authors listed. Highlight of prescribing information. http://www.accessdata. fda.gov/drugsatfda_docs/label/2008/021368s011lbl.pdf (date last accessed 01 December 2016).

35. Henrie AM, Nawarskas JJ, Anderson JR. Clinical utility of tadalafil in the treatment of pulmonary arterial hypertension: an evidence-based review. Core Evid 2015;10:99-109.

36. Park SH, O'Connor K, Sung R, et al. Comparison of healing process in open osteotomy model and closed fracture model. J Orthop Trauma 1999;13:114-120.

37. Perren SM. Evolution of the internal fixation of long bone fractures. The scientific basis of biological internal fixation: choosing a new balance between stability and biology. J Bone Joint Surg [Br]2002;84-B:1093-1110.

38. Gruenwald J. PDR for Herbal Medicines 2nd ed. Montvale, New Jersey Thompson: Medical Economics Co 2000:531-532.

39. Oliveira CH, Moraes ME, Moraes MO, et al. Clinical toxicology study of an herbal medicinal extract of Paullinia cupana, Trichilia catigua, Ptychopetalum olacoides and Zingiber officinale (Catuama) in healthy volunteers. Phytother Res 2005;19:54-57.

Funding Statement

- This study was supported by a grant from KLRM, LLC (Long Beach, California) and certain aspects were funded by the National Institute of Neurological Disorders and Stroke and the National Institute of General Medicine NINDS/ NIGMS SC1NS064611 (MGF), the National Institute on Minority Health and Health Disparities (NIMHD) 5U54MD00 7598-06 (MGF) and by NIMHD S21 MD000103 (MGF).

A. Neviaser reports consultancy fees received from DePuy Mitek which is not related to this article.

Author Contributions

- R. A. Rajfer: Study design, Data acquisition, Analysis, Interpretation, Drafting and critically revising the paper.

A. Kilic: Study design, Performed osteotomy.

A. S. Neviaser: Study design, Critically revising the paper.

- L. M. Schulte: Study design.

S. M. Hlaing: Data acquisition.

J. Landeros: Data acquisition.

- M. G. Ferrini: Study design, Data acquisition, Analysis, Interpretation, Drafting and critically revising the paper.

E. Ebramzadeh: Study design, Data acquisition, Analysis and interpretation of data S-H. Park: Study design, Data acquisition, Analysis, Interpretation, Critically revising the paper.

ICMJE Conflict of Interest

- R. Rajfer is related to two members of the board of KLRM LLC (the study sponsor), however, as this was a randomised study that was blinded to all investigators, any possibility of bias was removed.

(c) 2017 Park et al. This is an open-access article distributed under the terms of the Creative Commons Attributions licence (CC-BY-NC), which permits unrestricted use, 